\section{THE DAYLIGHT FIREBALL OF JULY 5 .}

VERY large meteors apparently exhibit a preference for the early evening hours. The fireball of March 28 last came at 7.48 p.m., and a great number of corroborative instances might be cited. The majority of these bodies travel with comparatively slow motion over extensive arcs, and are directed from radiant points in the western region of the heavens.

On Monday evening, July 5, at 8h. 3om., a few minutes after sunset, a splendid fireball passed from west to east in a long and nearly horizontal flight across the southern sky. The weather was generally clear over the south of England at the time, and thousands of observers were fortunate in catching a sight of the meteor as its nucleus disintegrated into a series of glistening balls strung on a fiery cord.

The spectacle was viewed by persons who sent in reports from Gloucestershire, Dorset, Hants, Essex, Somerset and Surrey, but the descriptions are rather indefinite owing to the conditions prevailing at the time. No stars were visible to which the path of the object might be referred. Yet, though daylight was so strong, the meteor brightly illumined the sky and attracted people to look upwards to ascertain the cause.

Mr. W. G. Wallace, of Broadstone, Dorset, writes that his sister saw the meteor in a S.S.E. direction, altitude about $30^{\circ}$. It disappeared over E. by S., altitude $20^{\circ}$. The object presented a brilliant mass of greenish-yellow light, moving slowly, and near the end of its flight it divided into two portions.

Mr. A. G. Pile, of Old Sodbury, Gloucestershire, observed the meteor moving from S.W. towards E. in from four to five seconds. It emitted a bluish-yellow flame, and looked like a large sky-rocket.

Mr. Dick, of Purley, Surrey, states that it quite lit up the sky, and travelled from S.W. to E. by S., about $20^{\circ}$ high. It split into two large fragments early in its flight. What specially struck him was the horizontal course, duration about $\mathrm{I}_{3}$ seconds.

Mr. W. J. Allen, of Thornbury, Glos., saw the bright light of the meteor traversing the sky in a horizontal direction from S.W. to E. When first noticed it apparently consisted of three electric balls, but at the end only two could be discerned.

Mrs. L. E. Butter, of Bishops Waltham, Hants, reports that her son, when sitting in the garden, called her to see a bright, comet-like appearance travelling from S.W. to eastward. There was a secondary head merging into the tail. The object finally burst like a rocket.

Mrs. H. I. W. May, of Chadwell Heath, Essex, relates that her daughters saw a brilliant star in the south going from west to east. While watching it the head divided into three stars and then disappeared.

At Bristol the meteor passed from W. of $S$. to E.S.E.; the angle of descent was slight, motion rather slow, and the nucleus consisted of two balls of fire, the leader being the largest. There was a profuse emission of sparks as the object sailed along, and the duration for the section of the flight which came under observation was six seconds.

Mr. E. W. Barlow writes that at Bournemouth the phenomenon was remarked by various people who could not, however, give exact particulars of the event. When passing due $S$. the altitude was $40^{\circ}$ to $45^{\circ}$, and the motion horizontal. The direction was from west to east.

I have been in correspondence with the various observers and elicited much further information, which has enabled me to derive the real path of the meteor. But the result may possibly require revision on the basis of additional records :-

$$
\text { No. } 2385 \text {, VOL. 95] }
$$

Fireball of July 5, 8h. 30m.

Radiant point $\ldots \quad \ldots \quad \ldots \quad \ldots \quad \ldots \quad 152^{\circ}-6^{\circ}$

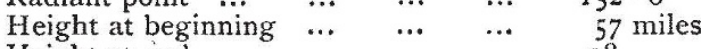
$\begin{array}{llllll}\text { Height at end } & \ldots & \ldots & \ldots & \ldots & 28,\end{array}$ Length of luminous path $\quad \ldots \quad \ldots . \quad 260 \quad$,

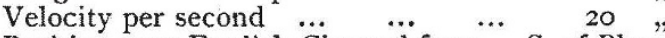
Position over English Channel from... S. of Plymouth

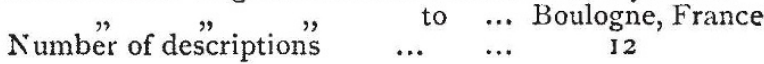

The radiant was near the $W$. by $S$. horizon, and its position must have been deflected several degrees towards the N.E. by the effect of zenithal attraction.

There is a special reason why so many fine meteors overtake the earth in nearly horizontal courses, and appear in the earlier hours of the night.

$$
\text { W. F. Denning. }
$$

\section{THE MATERIAL BASIS OF EVOLUTION.}

THE "Origin of Single Characters as Observed in Fossil and Living Animals" forms the subject of an illuminating essay in the American Naturalist for April, by Prof. H. F. Osborn. Since it contains some trenchant criticisms on recent attacks on the evolution theory, and on Darwin's work, it is likely to be much discussed in the immediate future.

The main purpose of his address is to insist on the importance of single characters, or "least characters," as indices of the trend of evolution rather than on the sum of the indefinite number of single characters which make up the individual. "In a sense," he remarks, "the species, subspecies, and variety, and even the individual, is not a zoological unit, whereas the ' character,' when narrowed down to the last point of divisibility, seems to be a unit ... and a very stable one, with certain distinctive powers, properties, and qualities of its own." This conception of the individual as a complex of separable and independently variable units represents a view which has been gaining ground for some time past.

But Prof. Osborn attempts to systematise this newer conception of the factors to be reckoned with in studying the elusive and complex phenomena associated with the transformation of animals. He distinguishes two aspects of this process-the study of the birth and development of proportional, and of numerical characters. Those of the first category he defines as universal and abundant; they are such as distinguish species and subspecies one from another, and may be germinal and therefore heritable, or merely somatic, due to environmental influences; while numerical characters, on the other hand, are solely germinal.

As numerical characters he cites the number of the teeth and of their cusps, the number of toes and of vertebræ, and so on, such being relatively stable characters which may be shared in common between a large number of species and genera.

That no hard and fast line can be drawn between "proportional" and "numerical" characters Prof. Osborn himself realises, for he uses as an illustration the reduction of the digits, as in the case of the evolution of the horse's foot. Hence it seems difficult to accept his dictum that proportional and numerical characters are due to a different series of direct causes. Rather they seem to be merely measures of degreequantitative and qualitative.

Towards the latter part of his essay he aims a blow at the Mendelians, and remarks that "If the student of genetics abandons the natural and the normal for the unnatural and the abnormal and sticks solely to his seed pan and his incubator, he is in danger of observing modes of origin and behaviour of characters which never have, and never will, occur in 